\title{
Manual Capsulorhexis in White Cataracts
}

\author{
Aliki Liaska* \\ Consultant Ophthalmic Surgeon, General Hospital of Lamia, Greece
}

*Corresponding author: Aliki Liaska, Consultant Ophthalmic Surgeon, General Hospital of Lamia, Greece.
Received Date: March 25, 2019

Published Date: April 17, 2019

\section{Opinion}

Even in the era of Femtosecond laser-assisted cataract surgery which has the advantage of creating a circular and optimally sized capsulotomy in cases of white cataract, manual capsulorhexis in white intumescent cataracts still holds a certain charm (and necessity as well, considering the difficulty of performing automated capsulorhexis in an anterior chamber with poor visibility due to leakage of liquified cortex through the initial capsulotomy). The incidence of residual adhesions was more in cases with release of white milky fluid affecting the creation of a free-floating capsulotomy [1]. The challenge is always to avoid the Argentinean flag sign, a severe complication consisting of a radial tearing during capsulorhexis in senile white cataracts. The tearing might extend beyond the equator [2]. It is always useful to remember the discrete types of white cataracts. It is the type 2, fluid-filled pearly white cataract or intumescent cataract that poses an extreme risk for this severe complication. Senile white cataracts-nomenclature (Figures 1-3)

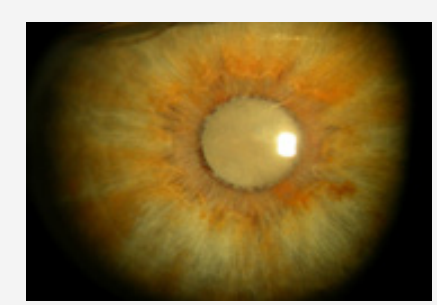

Figure 1: Fluid-free pearly white cataract or non-intumescent white cataract.

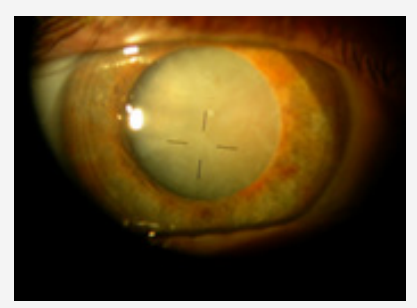

Figure 2: Fluid-filled pearly white cataract or intumescent cataract or mature white cataract.

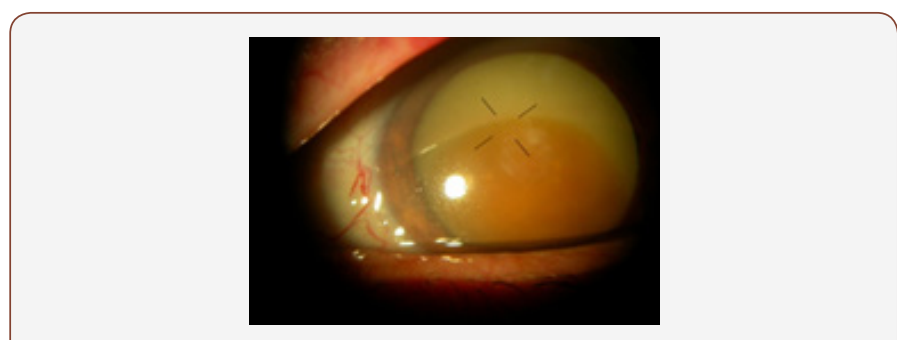

Figure 3: Hypermature white cataract or Morganian cataract.

Two-stage continuous curvilinear capsulorhexis [3], pressure on the nucleus after initial capsulorhexis to free liquified cortex [4], modified phaco capsulotomy technique [5], puncturing of the anterior capsule and gentle tipping of the nucleus to release fluid from the posterior intralenticular compartment [5] are some of the main techniques described to deal with the increased risk of radial tearing of the anterior capsule towards the equator at the initiation of capsulorhexis. We present a manual capsulorhexis technique for white, intumescent cataracts, which involves an initial intentional small capsulotomy to prevent the "argentinean flag" sign in phacoemulsification in intumescent lenses (a preliminary report of this technique was presented by Liaska et al, Free paper, XXXI ESCRS Congress Amsterdam, 2013) towards the direction of developing a strategy for consistently achieving continuous curvilinear capsulorhexis (CCC) [6,7].

Prospective, interventional study of 76 eyes of 76 patients with white, intumescent cataract operated with phacoemulsification in General Hospital of Lamia, from February 2014 to March 2017. The main steps of this technique consist of

- $\quad$ trypan blue injection to visualize the anterior capsule

- $\quad$ dispersive ocular viscoelastic device (OVD) injection in the anterior chamber to flatten the anterior lens surface

- $\quad$ quick small central radial capsulotomy (multiple small central cuts) performed with $27 \mathrm{G}$ capsulorhexis needle

- washout of the liquefied cortex 
- additionally, gentle pressure on the nucleus with the needle tip towards the posterior pole helps to release the fluid from posterior intralenticular compartment

- $\quad$ aspiration of as much fluid as possible

- continuous curvilinear capsulorrhexis (CCC) performed with capsulorhexis forceps using one of previously formed capsule flaps

The intentional multiple small radial cuts of the anterior capsule remain small and allow for the CCC. Even in cases where there was an imminent radial tear of the CCC, grasping another capsule flap made CCC feasible. There was no need for converting to extracapsular cataract extraction. The intentional small capsulotomy with multiple radial cuts as a preliminary stage in CCC in white intumescent cataracts relaxes the anterior capsule via neutralization of centrifugal forces and prevents large unexpected radial tears of the initial capsulotomy as well as the sudden radialization of the $\mathrm{CCC}$, thus providing a safer white cataract surgery.

\section{Acknowledgement}

None.

\section{Conflicts of Interest}

No Conflicts of Interest.

\section{References}

1. Titiyal JS, Kaur M, Singh A, Arora T, Sharma N (2016) Comparative evaluation of femtosecond laser-assisted cataract surgery and conventional phacoemulsification in white cataract. Clin Ophthalmol 10: 1357-1364.

2. Perrone D, Albertazzi R (2009) "Argentina Flag Sign", Video J Cataract Refract Surg Clinics (Sao Paulo). 64(4):309-212.

3. Kara-Junior N, De Santhiago MR, Kawakami A, Carricondo P, Hida (2009) Mini-rhexis for white intumescent cataracts. WT Clinics (Sao Paulo) 64(4): 309-312.

4. Figueiredo CG, Figueiredo J, Figueiredo GB (2012) Brazilian technique for prevention of the Argentinean flag sign in white cataract. J Cataract Refract Surg 38(9): 1531-1536.

5. Genç S, Güler E, Çakır H, Özertürk Y (2016) Intraoperative complications in intumescent cataract surgery using a phaco capsulotomy technique. Cataract Refract Surg 42(8): 1141-1145.

6. Dhingra D, Balyan M, Malhotra C, Rohilla V, Jakhar V, et al. (2018) A multipronged approach to prevent Argentinian flag sign in intumescent cataracts. Indian J Ophthalmol 66(9): 1304-1306.

7. Figueiredo CG Figueiredo J, Figueiredo GB (2012) Brazilian technique for prevention of the Argentinean flag sign in white cataract J Cataract Refract Surg 38(9): 1531-1536. 